\title{
Homologous series. Law or rule?
}

\author{
Valentine Suslov \\ Systems Biology Department \\ ICG SB RAS \\ Novosibirsk, Russia \\ valya@bionet.nsc.ru
}

\author{
Mikhail Ponomarenko \\ Systems Biology Department \\ ICG SB RAS \\ Novosibirsk, Russia \\ pon@bionen.nsc.ru
}

\author{
Dmitry Rasskazov \\ Systems Biology Department \\ ICG SB RAS \\ Novosibirsk, Russia \\ rassk@bionen.nsc.ru
}

\begin{abstract}
Despite the centenary, the status of Vavilov law of homologous series (LHS) remains uncertain. Unlike an empirical rule, a law may have no exceptions. Exceptions are indicative of having to abrogate or to ascertain the limits of the law's applicability, the latter being derivable from the ground on which the law stands. As the basis of the law neither the common ancestor heritage, nor the evolutionary enumeration of a limited space of possibilities satisfy this demand. We consider LHS as a consequence of the functional overlap of adaptive characters.
\end{abstract}

Keywords - Vavilov law of homologous series, promoter, TATA box, composition element, coadaptive substitutions, selfadaptive evolution.

\section{Background}

Similarity of homologous series (HS) is not weak, incomplete, or unexplored (in all cases, how much?) resemblance but an independent "third" similarity of characters caused by the principles of their organization ${ }^{1}$. HS does not require, does not exclude, but does not boil down to the identity (homology) and/or common function (analogy) of the characters [1]. The law has no exceptions, but only abrogate (a) and ascertain (b) limits of applicability. If $\mathbf{a}$, then the gaps in the HS are imaginary i.e. an observer error, a member of one HS fell into another, not derived from the first $(\alpha)$. If $\mathbf{b}$, then both the exception and the HS are equally inferred from one cause $(\beta)$. Otherwise, there is empirical rule, no law $(\gamma)$. Logically, $\alpha$ and $\beta$ are complementary, both oppose $\gamma:(\alpha \vee \beta) \wedge \neg \gamma$. For the first time, Cope spoke of HS as $(\alpha \vee \beta) \wedge \neg \gamma$, where a it's characters of genera without adaptive selection associated with morphogenetic dynamics which based on physicochemical laws; b it's characters of species that adaptive evolved only

1Hence obligate for Vavilov HSs character transgression [1]: in spite of a rapid divergence of phyla, transgressing characters retain the shared phenotypic aspects and the way the phenotype varies, while the convergence preserves only the phenotype.

2 The scheme that somehow underlies all modern evo-devo theories [3]. Since any hierarchy of characters automatically generates a modular organization of ontogenesis and parallelisms as a consequence, the problem of this scheme will not be HSs, but gaps which the scheme cannot deduce from an own foundation. For example [3] several gaps may be reduced by includ embrional forms in HS. But how deep can go down into embryos for it (not to mention that Vavilov used only postnatal characters in LHS [1])? The status of the law has only Stark-Zavarzin theorem [2], where the hierarchy is absolute, since it is given by the logical incompatibility of characters or their biochemistry that identic in adults and young.

3The same problem. Going down into embryogenesis and/or changing the complexity of characters by reducing them to sequences of biopolymers, an observer can arbitrarily get both homology (the postgenome homology crisis [5] foreseen by Meyen) and developmental hourglasses [4]. when a and the selection vector coincide [2]. Thus, any character of genera not only limited the space of possibilities (LSP) for characters of species, but also provided correlations between these characters ${ }^{2}$. However, the factual fuzziness of this characters' dichotomy has reduced the Cope laws to $\gamma$. In editions of Law of HS (LHS) of 1920 and especially 1922, Vavilov [1], having put forward many reasons, also reduced LHS to $\gamma$. In 1935 Vavilov [1] raises the question of $(\alpha \vee \beta) \wedge \neg \gamma$, for which he excludes from LHS the similarities whose reasons arise long before (i.e. popular today developmental hourglass and less popular common ancestor heritage $)^{3}$ or independently of this taxon (i.e. limitations by LSP or extreme physicochemical laws like Maupertuis principle). He continues to build only combinative (weak epistasis) ${ }^{4} \mathrm{HS}$, linking their genesis with centers of origin $(\mathrm{CO})^{5}$ [1]. But the schools of Filipchenko and Schmalhausen, paradigmatically claiming for LHS in fact returned to Cope (only characters hierarchy instead its dichotomy $)^{6}$. Is LHS the law? Are all HSs deducible from LHS?

\section{Materials and Methods}

We studied the phenotypic effect of mutations in the core and flanks of TATA boxes and composition elements (CEs) composed of TATA boxes with their flanking sequences overlapping other regulatory sites. The character under study was gene expression level: the service SNP_TATA_Comparator [2] discriminates between the TBP/TATA affinity contribution into the gene expression level and the regulatory site contribution for $\mathrm{CE}$ as well as between the contributions of the TATA box core and flanks.

4 Paradoxically ignoring correlations of characters that he himself appreciated. Including genetic maps, but not ploidy. For example, he built many HSs for separate flower's characters, but not one for flower's formula as a whole [1]. This formula, as known now, relies on a conservative gene network-automat.

5 Vavilov spoke about centers of origin [1], but neo- and paleo data do not confirm either the obligatory origin of a taxon in the center, or the supereffectiveness of the primary center for taxa's adaptive radiation [6,7]. Paradoxically, according to Vavilov, for LHS similarity forming it is necessary that the taxon invade into the region of maximum environmental diversity. That is disaccording to traditional theories [3] where similarity in environmental diversity is the least likely.

6According to Filipchenko [2], the hierarchy is determined by the time of the character appeared (from a common ancestor $[3,5]$ or nearest hourglass $[2,4]$ in the last version), to which the Modern Synthesis has added logical compatibility as Dobzhansky-Muller model [3]. According to Schmalhausen synthesis, this compatibility is regulated by feedbacks [2] and/or trade-offs [3]. 


\section{Results and Discussions}

Shown, that HSs appear due to coadaptive substitutions regardless of common ancestor: nonhomologous mutation in the TATA box mutually compensate for each other when there is a functional overlap (FO) between the flanks and the core. The same is as the TATA box has a FO with CE sites. Being dependent only on the level of diversity (in our case, on mutability) and the level of $F O$, this evolution will go both in a changeable and even in an unchangeable environments. But in both cases it should be a subpessimal environment. Finally, its own self-adaptive 7 trend is forming. The trend starts with the substitution of homology for being homologous and proceeds with a coordinated divergence of $\mathrm{HSs}^{8}$. During such a coordinated divergence, the bundle of closely related phyla carrying the character diverge for this character into bundles, in each of which phyla are differently related. Any bundle is a case of character transgression; any one-phylum bundle appears as a case of classic Darwinian bifurcation, an "exception" $\left(\beta_{1}\right)$ to LHS, but, on the other hand, many bundles this is any gap in each ( $\beta_{2}$, the main "exceptions" to LHS). A trend continues if self-adaptation in any bundle leads to the dominance of only one functional contribution to the gene expression level. For $\mathrm{CE}$ either the contribution of TBP/TATA affinity dominates, or the contribution of the regulatory site $^{9}$. Violation of the contribution symmetry forms the Harland scheme: major gene+gene (s)modifier(s) [8]. It is limit $\mathbf{b}$ to LHS. Since there are more than two modifiers and they are structurally separable, the aforementioned bundles coordinated divergence will increase, moreover, with all "exceptions", to which gaps in the number of modifier genes ${ }^{10}$, their contribution/effect and other its parameters are added $(\beta 3+\mathrm{k}) 11$. Bundles coordinated divergence will slow down with decrease in FO (weak epistasis, as Vavilov put it!) and will stop once FOs

${ }^{7}$ What makes self-adaptation be different from co-adaptation is a symmetry: a predator and a prey do not swap their roles, but a contributions to expression (providing that expression is underway), are interchangeable: in subpessimal environment not only decrease, but also increase in contribution coming from one site favors the fixation of the mutation that compensate for this effect. Evolution resembles Le Châtelier's principle ${ }^{[2]}$. In severe unchangeable environment a crucially necessary excess of the gene product blocks this symmetry; any gentle environment is a neutral [3] non-Vavilovian [1] evolution.

${ }^{8}$ In 1923 Sobolev was the first to observe it - with no comments left, though. The only case of divergence - seedshattering tips in rye, wheat and Aegilops plants - observed by Vavilov was mentioned in a comment, with no HSs created [1]!

${ }^{9}$ For TATA box, the contributors are either the core or the flanks, respectively. Since this pair of contributors is both functionally and structurally inseparable, the only possible trend here is the swing between the TATA+ and TATA-less promoters. The destruction of a pair is the death of the proximal promoter.

${ }^{10}$ Selection in favor of major gene means automatic selection in favor of modifier genes. However, among modifier genes, while their effect is evaluated in total, during this selection we, as a side result, will automatically receive advanced and lagging genes (the Abel's crossing of specializations). The contribution of the former is much larger, the contribution of the latter is significantly less than the average contribution for all modifier genes. Finally, have been banned; they may be banned for 4 reasons ( 3 identified for CEs): elimination of one of the characters, their emergent union 12 (high epistasis) or independent operation13. There are limits a to LHS.

\section{Conclusions}

LHS is a law. Not all HSs deducible from LHS ${ }^{14}$ but only HSs consisting of characters with FO.

\section{Acknowledgments}

The work supported by the budget project No. 0324-20190040-C01.

\section{References}

[1] N. Vavilov. "The law of homologous series in variation" Nauka. 1987. $260 \mathrm{p}$.

[2] V. Suslov, M. Ponomarenko, D. Rasskazov. "Homology and Vavilov's law of homologous series". http://mccmb.belozersky.msu.ru/2019/thesis/MCCMB2019/pa ges/author.utf8.html\#S.

[3] J. Herron, S. Freeman. "Evolutionary Analysis". Pearson. 2014. $864 \mathrm{p}$.

[4] I. Grosse. "Entropic Hourglass Patterns and the Emergence of Biodiversity". http://mccmb.belozersky.msu.ru/2019/thesis/MCCMB2019/pa ges/author.utf8.html\#G.

[5] J. Moore, P. Willmer. "Convergent evolution in invertebrates". Biol. Rev. Camb. Philos. Soc. vol. 72. pp. 1-60. 1997.

[6] N. Goncharov. "The centers of cultivated plants origin". Vestnik VOGiS. vol. 11. pp. 561-574. 2007.

[7] D. Begun, M. Nargolwalla, L. Kordos. "European Miocene hominids and the origin of the African ape and human clade". Evol Anthropol. vol. 21. pp. 10-23. 2012.

[8] S. Harland. "The genetical conceptions of the species". Biol. Rev. Camb. Philos. Soc. vol. 11. pp. 81-112. 1936.

lagging modifier genes must become neutral, pseudogenized, or even be eliminated if they cannot change function by Ohno model of Modern synthesis [3] $\left(\beta_{(4+\mathrm{k})}\right.$ "exception").

${ }^{11}$ Included by Vavilov to LHS in 1935 [1]. In many times used in synchronous criticism of LHS. Finally, it is equally excluded from LHS in Schmalhausen's and Modern syntheses as incompatible with the Cope foundation.

12 As in advanced Ohno models duplication-degradationsubfunctionalization in Modern synthesis [3]: each character is irreplaceable, but everyone works for common function. Vavilov LHS degenerate into Cope's rule.

${ }^{13}$ The fourth reason is Ukhtomsky dominante-like. Suppose the selection in favor of major gene supports only those modifier genes that enhance the phenotypic effect of major gene expression. Then the selection against antagonistic modifier genes is combined with two main contributions: for major gene and for synergistic modifier genes. Self-adaptation can contrasting or leveling these contributions. In the first case, we obtain an emergent character, in the last case, a death of Harland scheme after complete indistinguishability of these contributions.

${ }^{14}$ Besides the Cope's HSs it will be observed series of genetic maps (but not ploidy), where the violation of similarity is the destruction of the common ancestor heritage by transposons [3], whose activity is weakly associated with organismal characters with FO. 